\title{
MiR-145 reduces ADAM17 expression and inhibits in vitro migration and invasion of glioma cells
}

\author{
YONG LU $^{1}$, MICHAEL CHOPP ${ }^{1,2}$, XUGUANG ZHENG ${ }^{1}$, MARK KATAKOWSKI ${ }^{1}$, \\ BENJAMIN BULLER ${ }^{1}$ and FENG JIANG ${ }^{1}$ \\ ${ }^{1}$ Department of Neurology, Henry Ford Hospital, Detroit, MI; ${ }^{2}$ Department of Physics, \\ Oakland University, Rochester, MI, USA
}

Received May 10, 2012; Accepted June 1, 2012

DOI: $10.3892 / o r .2012 .2084$

\begin{abstract}
MicroRNAs are important regulators of gene expression and have been suggested to play a key role in tumorigenesis. In this study, we show that miR-145 is significantly downregulated in glioma cell lines compared to normal brain tissue and negatively regulates tumorigenesis. Restoration of miR-145 in glioma cells significantly reduced in vitro proliferation, migration and invasion. Also, overexpression of miR-145 reduced ADAM17 and EGFR expression. In addition, we tested the hypothesis that the miR-145-mediated suppression of cell proliferation, migration and invasion is, at least in part, due to silencing of ADAM17 and EGFR gene expression. Using luciferase reporters carrying the 3'-untranslated region of ADAM17 combined with western blotting, we identified ADAM17 as a direct target of miR-145. Collectively, these results suggest that as a tumor suppressor, miR-145 inhibits not only tumor proliferation, but also cell migration and invasion, and warrants further investigation.
\end{abstract}

\section{Introduction}

MicroRNAs (miRNAs) are short single-stranded nucleotide RNA molecules, which function as master regulators of gene expression by post-transcriptional modifications of target mRNAs (1). The pattern of regulation of gene expression is sequence-specific. MiRNAs bind to 3' untranslated regions (3'-UTRs) of mRNAs and then reduce the translation and/or stability of that mRNA, leading to a reduction in protein levels. Based on the unique feature of their targeting, miRNAs could have many targets (2), and, thus, control a large number of proteins.

MiRNAs may serve as either oncogenes or tumor suppressors $(3,4)$. In our study, the miR-145 is a tumor-suppressive miRNA (5) and its expression is low in different kinds of cancers. Mir-145 inhibits proliferation by targeting c-myc (6) and MUC1

Correspondence to: Dr Feng Jiang, Neurology Research, E\&R Building, Room 3056, Henry Ford Hospital, 2799 West Grand Boulevard, Detroit, MI 48202, USA

E-mail: fengj@neuro.hfh.edu

Key words: miR-145, ADAM17, EGFR, glioma, migration, invasion
(7). In addition, miR-145 can also promote differentiation and repress cell growth by repressing OCT4 (8).

ADAMs are best known as ectodomain sheddases, and their domains function as metalloproteases. The ADAM family is a Zn-dependent metalloproteinase (9,10). ADAM17 is an important member of the ADAM family and involved in proteolysis of collagen IV of the extracellular matrix and also the release from the cell surface of several integrins, suggesting that ADAM17 influences the invasive activity of different cells including glioma cells (11). ADAM17 is a primary upstream component for multiple EGFR pro-ligands $(12,13)$. EGFR binding with ligands subsequently activates MEK/ERK and PI3K/Akt pathways, which contribute to invasiveness and other malignant phenotypes (14).

In this study, the expression of miR-145 in glioma cells compared with normal brain tissue was studied by real-time RT-PCR. Proliferation, migration and invasion were examined to confirm the effects of miR-145 in glioma cells. The suppression of ADAM17 by miR-145 was confirmed by experiments using luciferase analysis and western blotting. We found that ectopic expression of miR-145 in U87 and U251n glioma cells caused decreased proliferation, migration and invasion with accompanying low protein expression of ADAM17 and EGFR. Our study provides direct evidence that miR-145 functions as an anti-oncogene in glioma cells and may be the target of therapies for glioma patients.

\section{Materials and methods}

Cells and miRNA transfection. U87-MG, U251n and T98G cells were obtained from the American Type Culture Collection (Manassas, VA, USA). HF66 cells were established by the Neurosurgical Department of Henry Ford Hospital. Cells and were maintained in Dulbecco's modified Eagle's medium (DMEM) supplemented with $10 \%$ fetal bovine serum (FBS). Total RNA from human frontal cortex brain tissue were obtained from Agilent Technologies (Santa Clara, CA, USA). Transfection of the miRIDIAN hsa-miR-145 miRNA mimic (Applied Biosystems), inactive (scrambled) control cel-mir-67 (Thermo Scientific Dharmacon, IL, USA), or pMIR-Report vectors was performed using Lipofectamine 2000 transfection reagent (Invitrogen, CA, USA) with $300 \mathrm{nmol}$ of miRNA or $1 \mu \mathrm{g} / \mathrm{ml}$ DNA plasmid, respectively. 

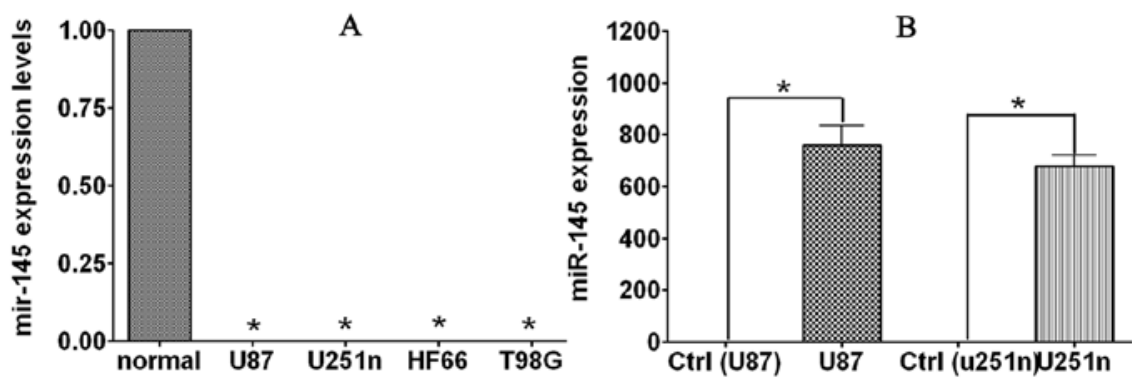

Figure 1. mir145 expression levels in glioma cell lines. (A) Total RNA was isolated from the normal brain tissue and glioma cells of U87, U251n, HF66, and T98G and real-time PCR was employed to analyze the expression of miR-145. The relative expression of miR-145 was expressed as the ratio of the expression level of normal brain tissue. " $\mathrm{P}<0.01$, as compared with normal brain tissues. (B) Glioma cells were transfected with miR-145 mimic for $72 \mathrm{~h}$, and then collected for real-time RT-PCR. The expression of miR-145 was significantly increased in U87 and U251n, as compared with non-transfected cells. "P<0.01, as compared to glioma cells without transfection of miR-145.

Western blot analysis and real-time RT-PCR. Western blot analysis was performed to detect ADAM17 (Abcam, MA, USA), EGFR, p-EGFR (Santa Cruz Biotechnology, Santa Cruz, CA, USA), and $\beta$-actin (Santa Cruz). Protein expression was measured $72 \mathrm{~h}$ after transfection. Protein concentration was quantified using a BCA protein assay kit (Pierce, Rockford, IL, USA). For quantitative analysis, densitometric measurements of three blots per group were averaged. Densitometry was performed with the MCID image analysis program (Cambridge, UK). The image density (1/intensity) of each band was normalized to $\beta$-actin, and experimental group band density was normalized to the band density of control. RT-PCR for miRNAs was performed using a miR-145 TaqMan MicroRNA assay. U6 miRNA was used as a house-keeping control. Each sample was tested in triplicate and relative gene expression was determined.

Invasion assay. Matrigel chambers (BD Biosciences) were used to determine the effect of miR-145 on invasiveness according to the manufacturer's instructions. Cells $\left(5 \times 10^{4}\right)$, after being transfected with miR-145 for $72 \mathrm{~h}$, were re-suspended in $500 \mu \mathrm{l}$ of serum-free medium and added to the upper chamber while the lower chamber was filled with $0.5 \mathrm{ml}$ of complete medium that served as a chemo-attractant. Cells were then incubated for $24 \mathrm{~h}$ at $37^{\circ} \mathrm{C}$. After removal of cells on the upper surface of the membrane, cells on the lower surface of the membrane were stained with CellTracker ${ }^{\mathrm{TM}}$ Green (Molecular Probes, Eugene, OR, USA) for $45 \mathrm{~min}$ and fixed in $4 \%$ formaldehyde. Nine fields of cells were counted randomly in each well under a fluorescent microscope at magnification $x 100$. All the experiments were done in duplicate and results were expressed as mean \pm SEM of three independent experiments.

Migration assay. After transfection, cells were seeded in 12-well plates in complete medium. When the cell confluence reached $\sim 90 \%$, at $72 \mathrm{~h}$ post-transfection, an artificial circle wound was made onto the monolayer with a 1-ml micropipette tip. The wound area was then examined after $24 \mathrm{~h}$ of incubation under an optical microscope at magnification x100. Photographs were taken and the cell migration ability was expressed by the gap closure.

Proliferation assay. Cell proliferation was measured using an ELISA kit (Roche Applied Science, Germany) according to the manufacturer's instructions. Briefly, $4 \times 10^{3}$ cells were plated to each well of a 96-well plate after transfection, and then cells were incubated with bromodeoxyuridine for $2 \mathrm{~h}$. The cells were subsequently fixed and incubated with $100 \mu 1$ of detection antibody conjugated with peroxidase and substrate, respectively. Light emission of the samples was measured in a microplate luminometer.

MirRNA luciferase assay. Position 416-422 of the ADAM17 3'-UTR is a predicted interaction position of miR-145. We cloned an 80-bp sequence containing the predicted binding site or a scrambled sequence downstream of the pMIR luciferase reporter to generate pMIR-ADAM17 and pMIR-mut-ADAM17 vectors, respectively. Luciferase assays were carried out in U87 cells. First, cells were co-transfected with appropriate plasmids with either hsa-miR-145 miRNA mimic or control cel-mir-67 mimic in 12-well plates. Then, the cells were harvested and lysed for luciferase assay $24 \mathrm{~h}$ after transfection. Luciferase assays were performed using a luciferase assay kit (Promega) according to the manufacturer's instructions.

Statistical analysis. Data are presented as mean and standard error (mean \pm SEM). Statistical significance was analyzed by one-way ANOVA using the GraphPad Prism software (version 4.0). P-value smaller than $0.05(\mathrm{P}<0.05)$ was considered significant.

\section{Results}

Expression profile of miR-145 in glioma cells. A growing body of studies report that miR-145 is downregulated in cancers $(5,15-19)$. However, the expression of miR-145 in glioma has not been well documented $(20,21)$. We sought to identify the role of miR-145 in human glioma cells. We compared the expression levels of miR-145 in glioma cell lines with total RNA from frontal cortex using real-time RT-PCR. As shown in the Fig. 1A, miR-145 expression levels were significantly decreased in tumor cell lines compared to total RNA from normal brain tissues. Expression of miR-145 in U87, U251n, T98G and HF66 glioma cell lines $0.113,0.002,0.045$ and $0.002 \%$ of normal brain tissue. The reduced expression of miR-145 in glioma cells suggests that miR-145 is a potential target in glioma therapy.

MiR-145 overexpression inhibits proliferation of glioma cells. To test the effect of miR-145 on cell growth, we used miR-145 

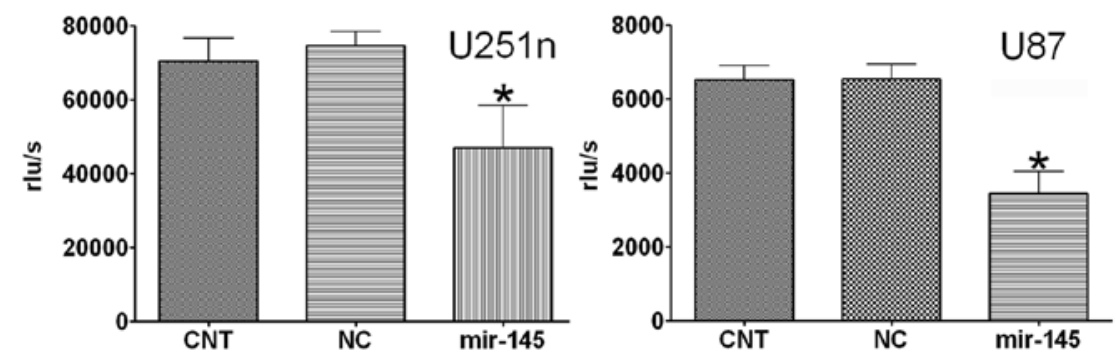

Figure 2. Overexpression of miR-145 reduces glioam cell proliferation in vitro. Glioma cells were transfected with miR-145 and negative control. After incubation for $72 \mathrm{~h}$, cell proliferation rates were analyzed by BrdU assay. ${ }^{\text {P }}<0.01$, as compared to control and negative control.

A
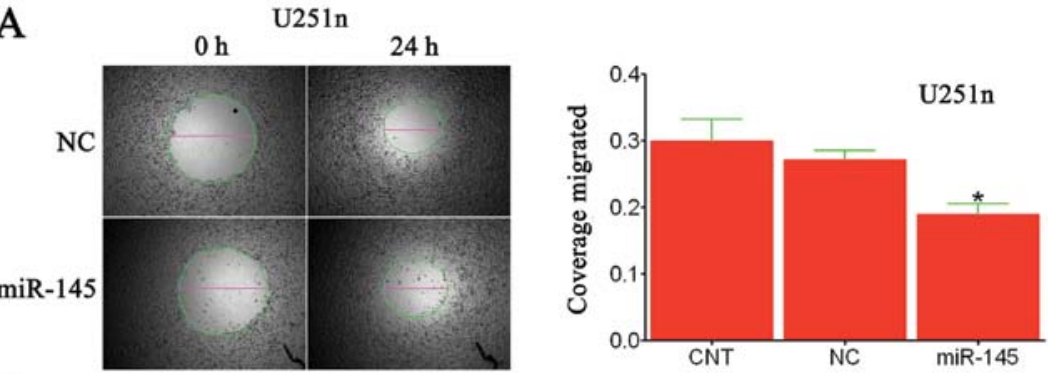

B
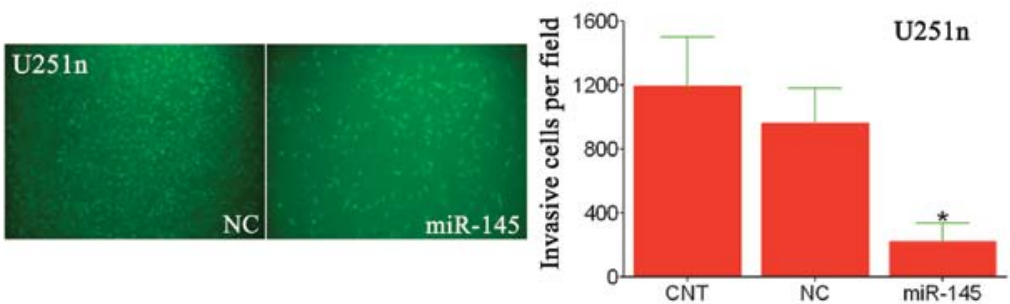

$\mathrm{C}$
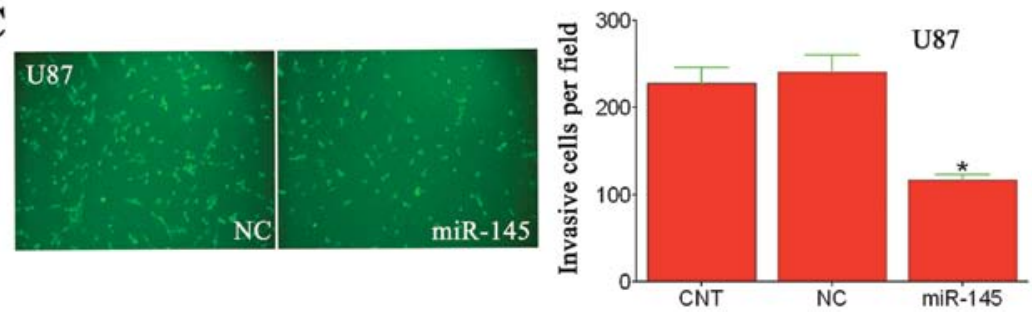

Figure 3. Effect of miR-145 overexpression on cell migration and invasion in U251n and U87. (A) Wound healing assay was performed to evaluate the effects of miR-145 on glioma cell migration. U251n cells were transfected with miR-145 at a final concentration of $300 \mathrm{nM}$. When the cell confluence reached $\sim 90 \%$ at $72 \mathrm{~h}$ post-transfection, an artificial homogeneous wound was made as described in Materials and methods. The experiments were preformed in triplicate and the representative images of photographs at 0 and $24 \mathrm{~h}$ post-wounding are shown at magnification $\mathrm{x} 100$. $^{*} \mathrm{P}<0.01$, as compared to control and negative control. (B) Effect of miR-145 overexpression on glioma cell invasion. Glioma cells transfected with miR-145 or negative control were plated on Matrigel-coated membranes in the upper chamber of transwells. After incubation for $24 \mathrm{~h}$, non-invading cells on the upper surface of the membrane were removed and the invasive cells on the lower surface were stained with cell tracker. The stained invasive cells were photographed under a fluorescence microscope (magnification $\mathrm{x} 200$ ). ${ }^{*} \mathrm{P}<0.01$, as compared to control and negative control. (C) To confirm the effect of miR-145 overexpression on glioma cells invasion, we employed the cell line U87. U87 cells were also transfected with miR-145 or negative control, and then analyzed with Matrigel-coated membranes chamber invasion assay. ${ }^{*} \mathrm{P}<0.01$, as compared to control and negative control.

precursor microRNA to infect human glioma U251n and U87 cells. After transfection, miR-145 levels were increased in both cell lines, indicating that enhancement was due to the introduction of precursor miR-145 (Fig. 1B). As demonstrated by BrdU assay, overexpression of miR-145 significantly reduced cell proliferation by $>40 \%$ in U87 and U251n as compared to cells transfected with control (Fig. 2). These data suggest miR-145 has anti-proliferative effects on $\mathrm{U} 87$ and $\mathrm{U} 251 \mathrm{n}$ in vitro.

Ectopic expression of miR-145 inhibits glioma cell migration and invasion. Previous studies reported that miR-145 decreases inva- sion and migration of breast cancer cells in vitro and in vivo (7). ADAM17 and EGFR have been reported to play important roles in glioma, and overexpression of ADAM17 promotes glioma invasiveness $(22,23)$. To determine if miR-145 had a similar effect on glioma cells, we employed a wound healing migration assay and a Matrigel invasion chamber assay. Seventy-two hours after transfection with miR-145 or scrambled-miRNA, U87 and U251n cells were seeded into the upper chamber, and then cells that invaded through the extracellular matrix after $24 \mathrm{~h}$ were imaged and counted (Fig. 3B and C). In both cell lines, miR-145 significantly decreased the number of cells that invaded 


\section{A H. Sapiens ADAM 17, 3' UTR 5' ...UUUAUUUGUGAUGACAACUGGAA... |||||| \\ Has-miR-145 $\quad 3^{\prime} \quad$ UCCCUAAGGACCCUUUUGACCUG}

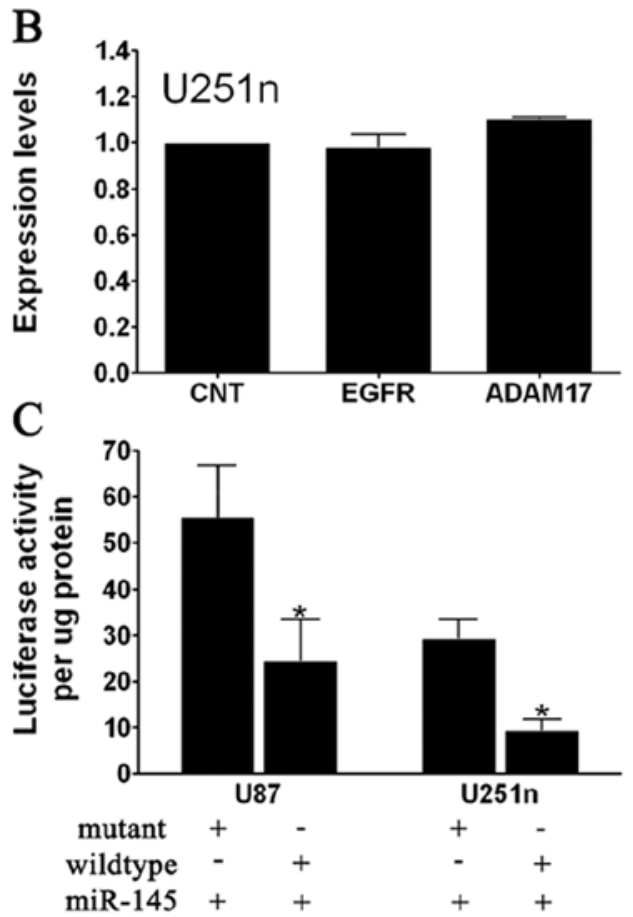

$\mathrm{D}$

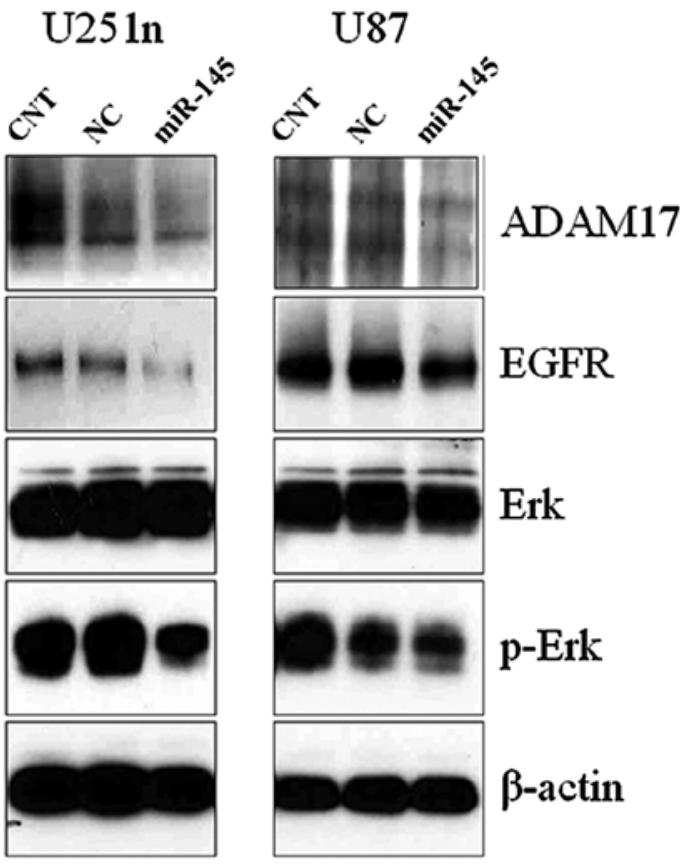

Figure 4. ADAM17 is a predicted target of miR-145 for post-transcriptional repression. (A) The miR-145 seed sequences and their predicted binding sites in the ADAM17 3'-UTR are shown. The highlighted region representing the binding site was predicted by Targetscan. (B) The mRNA expression of ADAM17 and EGFR in glioma cells. Overexpression of miR-145 did not decrease the mRNA expression of EGFR and ADAM17. (C) MiR-145 targets the predicted binding site within the ADAM17 mRNA 3'-UTR, decreasing luciferase in U87 and U251n cells transfected with a luciferase reporter vector containing the predicted binding site. Mutant-ADAM17, vector containing mutated binding site; wild-type-ADAM17, vector containing predicted binding site. "P $<0.01$ compared to U87 or U251n transfected with vector containing a mutated ADAM17 site and miR-145. (D) The protein expression of ADAM17 and EGFR in U251n cells. Western blot analysis was employed to test the expression of ADAM17 and EGFR in glioma cells. $\beta$-actin was used as an internal standard.

compared to controls. Similarly, using a wound healing assay, we examined the effect of miR-145 on U251n cell migration (Fig. 3A). Here, we found miR-145 inhibited migration of U251n glioma cell migration, as compared with scrambled-miRNA transfected control groups. Taken together, these data indicate that miR-145 serves as a regulatory molecule involved in cell migration and invasion in vitro.

ADAM17 and EGFR as targets of miR-145 for post-transcriptional repression. Given that miR-145 reduces migration and invasion of U251n and U87 glioma cells, we then sought to identify the target genes of miR-145 using the online miRNA target prediction programs Targetscan and Pictar (Fig. 4A). Approximately 150 targets of miR-145 were predicted from these programs. ADAM17 was of particular interest because our previous studies revealed that its expression level was upregulated in glioma specimens. ADAM17 overexpression contributes to glioma progression (23). It was recently reported that miR-145 inhibits expression of EGFR and reduces cell growth of lung cancer cells (24).

To test whether miR-145 suppresses the expression of ADAM17 and EGFR at protein level in glioma, we performed Western blot analysis. Employing a 'scrambled' cel-mir-67 or miR-145 mimic, we found that ADAM17 and EGFR protein expression were significantly reduced by miR-145 compared to miRNA controls in both cell lines (Fig. 4D). However, overexpression of miR-145 did not change mRNA expression of ADAM17 and EGFR (Fig. 4B). These data suggest that miR-145 targets ADAM17 and EGFR by post-transcriptional repression.

ADAM17 is a primary sheddase for multiple EGFR proligands $(25,26)$. EGFR is an important mediator responsible for the invasiveness of malignant gliomas $(14,27)$. EGFR ligand binding results in receptor self-dimerization, autophosphorylation and subsequent activation of downstream Ras/MAPK/ ERK signaling pathways, which contribute to the malignant phenotype (28). As reduced protein expression of ADAM17 and EGFR by miR-145, Western blot analysis was also employed to determine the expression of downstream signaling protein Erk/p-Erk. Compared to controls, miR-145 has no effect on Erk, but significantly decreased $\mathrm{p}$-Erk expression. These data indicate that miR-145 may reduce migration and invasion through the ADAM17/EGFR/Erk/p-Erk signaling pathway.

To further confirm that the effects observed above are ascribed to the specific interaction between miR-145 and the binding sites for miR-145 in the 3'-UTR of ADAM17, we performed a Luciferase activity assay. As shown in Fig. 4C, miR-145 suppressed $>40$ and $50 \%$ activity, respectively, of Luc-ADAM17-UTR (wild-type) in U87 and U251n compared 
with mutant ADAM17 3'-UTR, which suggest that miR-145 directly targets the 3'-UTR of ADAM17 mRNA.

\section{Discussion}

In the present study we detected the miR-145 expression level in U87, U251n, T98G and HF66 human glioma cell lines, and found a significant reduction of miR-145 in human glioma cell lines compared to total RNA of frontal cortex. The expression level of miR-145 negatively regulates glioma malignancy. Ectopic expression of miR-145 decreased proliferation, migration, and invasion of glioma cells. These data suggest that miR-145 plays a critical role in glioma development, and it may function as an anti-tumor factor in glioma cells.

Our studies indicate that ADAM17 is a target gene of miR-145, and several lines of evidence support the direct interaction between miR-145 and ADAM17: i) the 3'-UTR of ADAM17 contains a binding site for miR-145 with significant seed match; ii) miR-145 suppresses the activity of a luciferase reporter with the 3'-UTR of ADAM17 mRNA; iii) miR-145 represses ADAM17 expression at protein level; (4) our previous studies have shown that ADAM17 knockdown by siRNAs resulted in a decreased glioma cell proliferation, migration and invasiveness. We therefore, the first time, identify miR-145 as directly regulating ADAM17.

ADAM17 expression is elevated in different cancers and is associated with cancer progression $(29,30)$. High levels of ADAM17 have been reported in a variety of cell lines and clinical tissue samples, including breast, colon, prostate and glioblastoma (23,31-33). Our previous study showed that high level expression of ADAM17 was found in high grade glioma samples. Knockdown of ADAM17 inhibits breast cancer and glioma cell proliferation and invasion $(23,31)$. Ectopic expression of ADAM17 induced tumorigenicity of cortical astrocyte cell line (34) and promotes both breast and glioma cell malignant phenotypes $(23,31)$. In our present study, we demonstrated that miR-145 is an important regulator of ADAM17, and directly binds the 3'-UTR of ADAM17 mRNA in glioma cells. Thus, the reduction of miR-145 in glioma cells yields an increased expression of ADAM17.

One of the characteristics of glioma is invasiveness. The EGFR is over-expressed in $\sim 50-60 \%$ of gliomas (35), and EGFR increases with malignancy grade, and is required for maintenance of glioma growth (36). EGFR serves as an aggressive agonist of glioma invasion (37). In our study, transfection of glioma cells with miR-145 resulted in decreased protein expression of EGFR, which may be partly responsible for the miR-145 induced decrease of glioma cell invasion, and consistent with a recent report that miR-145 decreased EGFR expression and proliferation in lung cancers (38). These data suggest that miR-145 may also target the EGFR gene. ADAM17 is involved in the ectodomain shedding of multiple membrane-bound ligands and cytokines, implicated in diverse biological processes, including growth and inflammation $(23,39)$. Interestingly, ADAM17 has recently been identified as the primary sheddase for multiple EGFR pro-ligands. EGFR ligand-binding results in receptor self-dimerization, autophosphorylation and subsequent activation of downstream Ras/ MAPK/ERK signaling pathways $(28,40)$. Taken together with reduced p-Erk expression, miR-145 transfection may decrease glioma cell migration and invasion through ADAM17/EGFR/ ERK signaling pathway.

In conclusion, our data suggested that miR-145 plays a key role in the malignancy of glioma cells possibly by direct regulation of ADAM17 protein expression, which affects glioma cell proliferation, migration and invasion. However, further study is needed to determine if ADAM17 activity is influenced by miR-145 in vivo in glioma.

\section{Acknowledgements}

This work was supported by NIH grant RO1 CA129446.

\section{References}

1. Yi R and Fuchs E: MicroRNAs and their roles in mammalian stem cells. J Cell Sci 124: 1775-1783, 2011.

2. Gusev Y: Computational methods for analysis of cellular functions and pathways collectively targeted by differentially expressed microRNA. Methods 44: 61-72, 2008.

3. Garzon R, Calin GA and Croce CM: MicroRNAs in cancer. Annu Rev Med 60: 167-179, 2009.

4. Shenouda SK and Alahari SK: MicroRNA function in cancer: oncogene or a tumor suppressor? Cancer Metastasis Rev 28: 369-378, 2009.

5. Akao Y, Nakagawa Y and Naoe T: MicroRNA-143 and -145 in colon cancer. DNA Cell Biol 26: 311-320, 2007.

6. Chen Z, Zeng H, Guo Y, et al: miRNA-145 inhibits non-small cell lung cancer cell proliferation by targeting c-Myc. J Exp Clin Cancer Res 29: 151, 2010.

7. Sachdeva M and Mo YY: MicroRNA-145 suppresses cell invasion and metastasis by directly targeting mucin 1 . Cancer Res 70: 378-387, 2010.

8. Wu Y, Liu S, Xin H, et al: Up-regulation of microRNA-145 promotes differentiation by repressing OCT4 in human endometrial adenocarcinoma cells. Cancer 117: 3989-3998, 2011.

9. Lu X, Lu D, Scully M and Kakkar V: ADAM proteins - therapeutic potential in cancer. Curr Cancer Drug Targets 8: 720-732, 2008.

10. Kheradmand F and Werb Z: Shedding light on sheddases: role in growth and development. Bioessays 24: 8-12, 2002.

11. Wildeboer D, Naus S, Amy Sang QX, Bartsch JW and Pagenstecher A: Metalloproteinase disintegrins ADAM8 and ADAM19 are highly regulated in human primary brain tumors and their expression levels and activities are associated with invasiveness. J Neuropathol Exp Neurol 65: 516-527, 2006.

12. Zheng X, Jiang F, Katakowski M, et al: Inhibition of ADAM17 reduces hypoxia-induced brain tumor cell invasiveness. Cancer Sci 98: 674-684, 2007.

13. Zheng X, Jiang F, Katakowski M, et al: Sensitization of cerebral tissue in nude mice with photodynamic therapy induces ADAM17/TACE and promotes glioma cell invasion. Cancer Lett 265: 177-187, 2008.

14. Tsatas D, Kanagasundaram V, Kaye A and Novak U: EGF receptor modifies cellular responses to hyaluronan in glioblastoma cell lines. J Clin Neurosci 9: 282-288, 2002.

15. Gregersen LH, Jacobsen AB, Frankel LB, Wen J, Krogh A and Lund AH: MicroRNA-145 targets YES and STAT1 in colon cancer cells. PLoS One 5: e8836, 2010.

16. Ozen M, Creighton CJ, Ozdemir M and Ittmann M: Widespread deregulation of microRNA expression in human prostate cancer. Oncogene 27: 1788-1793, 2008.

17. Takagi T, Iio A, Nakagawa $\mathrm{Y}$, Naoe T, Tanigawa N and Akao $\mathrm{Y}$ : Decreased expression of microRNA-143 and -145 in human gastric cancers. Oncology 77: 12-21, 2009.

18. Akao Y, Nakagawa Y, Kitade Y, Kinoshita T and Naoe T: Downregulation of microRNAs-143 and -145 in B-cell malignancies. Cancer Sci 98: 1914-1920, 2007.

19. Ichimi T, Enokida H, Okuno Y, et al: Identification of novel microRNA targets based on microRNA signatures in bladder cancer. Int J Cancer 125: 345-352, 2009.

20. Fischer L, Hummel M, Korfel A, Lenze D, Joehrens K and Thiel E: Differential micro-RNA expression in primary CNS and nodal diffuse large B-cell lymphomas. Neurooncology 13: 1090-1098, 2011. 
21. Fang X, Yoon JG, Li L, et al: The SOX2 response program in glioblastoma multiforme: an integrated ChIP-seq, expression microarray, and microRNA analysis. BMC Genomics 12: 11, 2011.

22. Lo HW: EGFR-targeted therapy in malignant glioma: novel aspects and mechanisms of drug resistance. Curr Mol Pharmacol 3: 37-52, 2010.

23. Zheng X, Jiang F, Katakowski M, Lu Y and Chopp M: ADAM17 promotes glioma cell malignant phenotype. Mol Carcinog 51: 150-164, 2012.

24. Cho WC, Chow AS and Au JS: Restoration of tumour suppressor hsa-miR-145 inhibits cancer cell growth in lung adenocarcinoma patients with epidermal growth factor receptor mutation. Eur J Cancer 45: 2197-2206, 2009.

25. Hart S,Fischer OM and Ullrich A: Cannabinoids induce cancer cell proliferation via tumor necrosis factor alpha-converting enzyme (TACE/ADAM17)-mediated transactivation of the epidermal growth factor receptor. Cancer Res 64: 1943-1950, 2004.

26. Lee DC, Sunnarborg SW, Hinkle CL, et al: TACE/ADAM17 processing of EGFR ligands indicates a role as a physiological convertase. Ann NY Acad Sci 995: 22-38, 2003.

27. Laerum OD, Nygaar SJ, Steine S, et al: Invasiveness in vitro and biological markers in human primary glioblastomas. J Neurooncol 54: $1-8,2001$

28. Wells A: EGF receptor. Int J Biochem Cell Biol 31: 637-643, 1999.

29. Kornfeld JW, Meder S, Wohlberg M, et al: Overexpression of TACE and TIMP3 mRNA in head and neck cancer: association with tumour development and progression. Br J Cancer 104: 138-145, 2011.

30. Narita D, Seclaman E, Ursoniu S and Anghel A: Increased expression of ADAM12 and ADAM17 genes in laser-capture microdissected breast cancers and correlations with clinical and pathological characteristics. Acta Histochem 114: 131-139, 2012.
31. Zheng X, Jiang F, Katakowski M, Zhang ZG, Lu QE and Chopp M: ADAM17 promotes breast cancer cell malignant phenotype through EGFR-PI3K-AKT activation. Cancer Biol Ther 8: 1045-1054, 2009.

32. Blanchot-Jossic F, Jarry A, Masson D, et al: Up-regulated expression of ADAM17 in human colon carcinoma: co-expression with EGFR in neoplastic and endothelial cells. J Pathol 207: 156-163, 2005.

33. Lin P, Sun X, Feng T, et al: ADAM17 regulates prostate cancer cell proliferation through mediating cell cycle progression by EGFR/PI3K/AKT pathway. Mol Cell Biochem 359: 235-243, 2012.

34. Katakowski M, Jiang F, Zheng X, Gutierrez JA, Szalad A and Chopp M: Tumorigenicity of cortical astrocyte cell line induced by the protease ADAM17. Cancer Sci 100: 1597-1604, 2009.

35. Heimberger AB, Hlatky R, Suki D, et al: Prognostic effect of epidermal growth factor receptor and EGFRvIII in glioblastoma multiforme patients. Clin Cancer Res 11: 1462-1466, 2005.

36. Mukasa A, Wykosky J, Ligon KL, Chin L, Cavenee WK and Furnari F: Mutant EGFR is required for maintenance of glioma growth in vivo, and its ablation leads to escape from receptor dependence. Proc Natl Acad Sci USA 107: 2616-2621, 2010.

37. Tysnes BB, Haugland HK and Bjerkvig R: Epidermal growth factor and laminin receptors contribute to migratory and invasive properties of gliomas. Invasion Metastasis 17: 270-280, 1997.

38. Cho WC, Chow AS and Au JS: MiR-145 inhibits cell proliferation of human lung adenocarcinoma by targeting EGFR and NUDT1. RNA Biol 8: 125-131, 2011.

39. Franovic A, Robert I, Smith K, et al: Multiple acquired renal carcinoma tumor capabilities abolished upon silencing of ADAM17. Cancer Res 66: 8083-8090, 2006.

40. Lal A, Glazer CA, Martinson HM, et al: Mutant epidermal growth factor receptor up-regulates molecular effectors of tumor invasion. Cancer Res 62: 3335-3339, 2002. 